\title{
Société Suisse de radio-oncologie (SSRO)
}

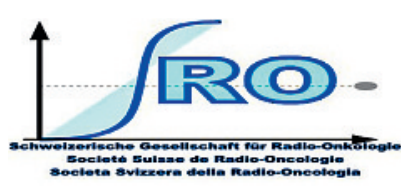

Günther Gruber

Président de la SSRO
Correspondance:

Dr Günther Gruber, privat-docent Clinique Hirslanden

Institut de radiothérapie

Witellikerstrasse 40

CH-8032 Zurich

Tél. 0443872550

guenther.gruber@hirslanden.ch

\section{Qu'est-ce que la radio-oncologie?}

La radio-oncologie/radiothérapie porte sur l'application médicale des rayons ionisants tant pour soigner les maladies que pour calmer la douleur. La plupart des patients suivent une radiothérapie pour traiter une tumeur maligne. Plusieurs groupes de spécialistes sont impliqués dans les traitements par irradiation. Outre le médecin, il s'agit du physicien médical, des technicien-nes en radiologie médicale, du personnel soignant ainsi que des techniciens et des collaborateurs du secrétariat.

\section{La Société Suisse de radio-oncologie (SSRO)}

En règle générale, les médecins spécialistes sont membres de la SSRO. La formation postgraduée et continue est l'un des domaines d'activité-clé du Comité qui compte actuellement cinq membres. A ce sujet et concernant d'autres points essentiels pour la radio-oncologie/radiothérapie (p.ex. la radioprotection et l'assurance-qualité), la SSRO travaille en étroite collaboration avec la Scientific Association of Swiss Radiation Oncology (SASRO) et la Société Suisse de Radiobiologie et de Physique Médicale (SSRPM). Notre discipline compte actuellement 15 établissements de formation postgraduée, dont 10 en catégorie A (reconnus pour 4 ans) et 5 en catégorie B (reconnus pour 2 ans). La formation postgraduée en vue de l'obtention du titre de spécialiste dure 6 ans.

\section{Quelques chiffres}

La SSRO est une petite société de discipline médicale par rapport à d'autres. Au cours des 15 dernières années, seuls 82 titres de spécialistes ont été décernés par la FMH, et ce bien que le nombre de patients traités chaque année dans ce domaine ne cesse d'augmenter. Les données statistiques pour la radio-oncologie en Suisse le montrent: en 2007, environ 20000 patients ont été traités en Suisse, dont environ 800 pour une affection bénigne (p. ex. épicondylite, arthrose), ce qui correspond à une augmentation de $15 \%$ par rapport à 2004. L'accroissement de la charge de travail est également visible en 2007 où un spécialiste en radio-oncologie traitait 236 patients contre 202 en 2004. En comparaison, ce nombre s'est élevé aux Pays-Bas à 217 patients par médecin durant la même année. En outre, le nombre

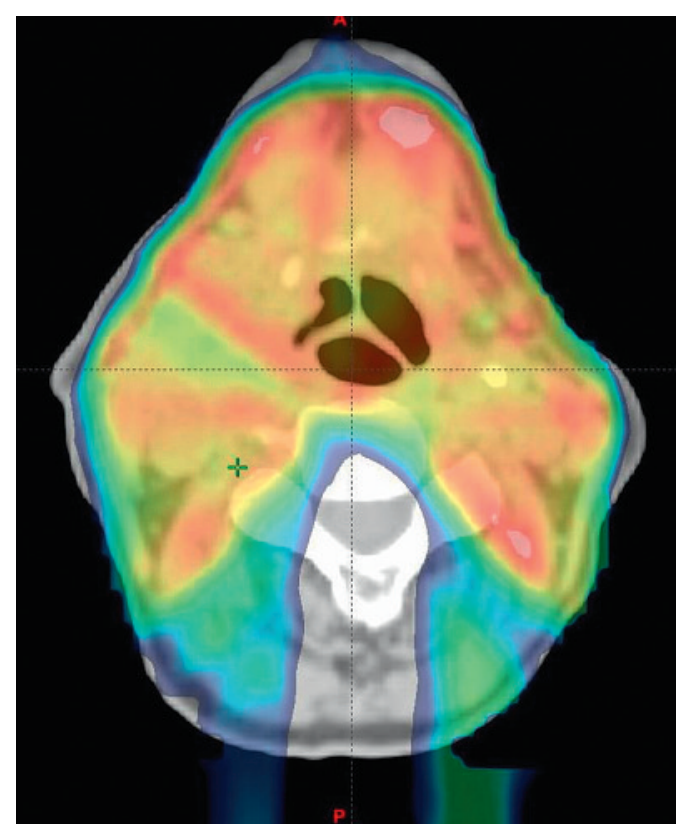

RTIM: répartition des doses.

de séances de thérapie à très haute tension (2007: 371804 ) et celui des champs d'irradiation (2007: 1333533) ont augmenté de plus de $40 \%$, un chiffre impressionnant et disproportionné au vu du nombre de patients, mais qui rend compte de la complexité croissante des traitements. En réalité, le recours à la radiochirurgie a augmenté de $21 \%$, celui à la brachythérapie (= traitement de courte distance) de $86 \%$ et celui à la radiothérapie à intensité modulée (RTIM) de 404\%! Durant le même laps de temps, on a constaté un léger recul du nombre de médecins pratiquant la radiothérapie ( $-6 \%$ environ) et certains centres ont des difficultés à pourvoir leurs postes de formation.

\section{Perspectives}

L'une des principales préoccupations de notre société consiste dès lors à rendre la radio-oncologie plus attrayante aux yeux des jeunes médecins. L'utilisation de nouvelles procédures d'imagerie dans le déroulement du traitement (p.ex. diffusion d'images, radiotomographie associée à un accélérateur linéaire), l'augmentation de techniques d'applications particulières et l'intégration de la radiothérapie dans un concept thérapeutique multimodal devraient accroître l'attrait de la discipline. 